\title{
Remedy to overestimation of classical interval analysis: Analysis of beams with uncertain boundary conditions
}

\author{
Isaac Elishakoff ${ }^{\mathrm{a}, *}$ and Clément Soret ${ }^{\mathrm{b}}$ \\ ${ }^{a}$ Department of Ocean and Mechanical Engineering, Florida Atlantic University, Boca Raton, FL, USA \\ ${ }^{\mathrm{b}}$ Institut Français de Mécanique Avancée, Aubière, France
}

Received 21 July 2011

Revised 13 December 2011

\begin{abstract}
This study investigates both the static and dynamic behaviors for the uniform beam supported at its ends by rotational supports. The stiffness coefficients of these supports are treated as interval variables. It is first demonstrated on the static problem that the classical interval analysis may lead to overestimation of the response. The paper introduces a remedy for this "ill" of the classical interval analysis. The artificial interval parameter is introduced and the extrema of the static displacements and natural frequencies are determined. Finally, the hybrid case of uncertain boundary conditions and uncertain mass density is treated with attendant interesting results.
\end{abstract}

Keywords: Interval analysis, beam vibrations, rotational springs, parametrization technique

\section{Introduction}

Modeling of boundary conditions is an important part of engineering analysis. The idealized boundary conditions, as a simply supported end, or as a clamped end, seldom appear in the real engineering practice.

Therefore, one has to introduce springs, either translational or rotational, in order to accurately represent realistic boundary conditions.

There are number of studies that deal with static behavior and vibrations of beams with springs at their ends. These include papers by Laura et al. [1], Gürgöze [2], Alvarez et al. [3] and others. It must be noted that such studies are of paramount importance for identifying true boundary conditions. (see, e.g. Sweet et al. [4]). These works treat the spring coefficients as deterministic constants.

However, in an ensemble of macroscopically identical beams there is a scatter in the elastic springs' coefficients and thus, in boundary conditions that these springs represent. It appears that it is a must to incorporate uncertainty in analysis of structure with spring supports.

Several analyses of this kind have been reported in literature. For example, Ben-Haim and Natke [5] and Elishakoff and Fang [6] utilized convex modeling to diagnose load modifications in boundary conditions. Cherki et al. [7] utilized fuzzy sets based analysis. Zalewski et al. [8] employed interval analysis.

In this study we utilize modified interval analysis that yields accurate lower and upper bounds without excessive numerical effort.

\footnotetext{
* Corresponding author: Isaac Elishakoff, Department of Ocean and Mechanical Engineering, Florida Atlantic University, Boca Raton, FL33431-0991, USA. Tel.: +1 561297 2729; Fax: +1 561297 2825; E-mail: elishako@fau.edu.
} 


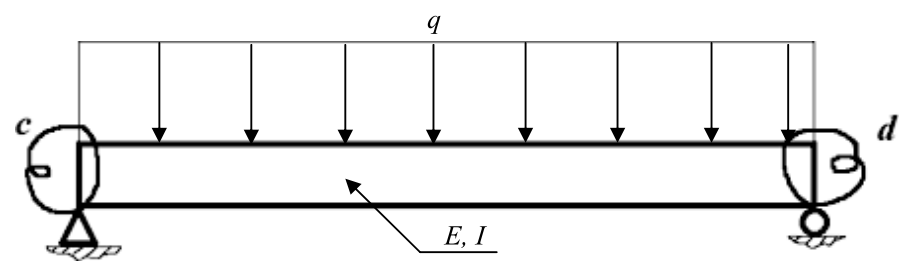

Fig. 1. Uniform beam with rotational springs at its ends under uniformly distributed load.

\section{Basic equations}

We first consider a uniform beam with rotational springs at the ends as shown in Fig. 1.

The beam is pinned at its ends; likewise, it has rotational springs at each end. The governing differential Eq. for a uniform beam under a distributed load is

$$
E I \frac{d w^{4}}{d x^{4}}=-q
$$

where $E$ is the Young's modulus of elasticity, $I$ is the area moment of inertia, $q$ is the load and $c$ and $d$ are the torsional rigidities of the spring.

Following Rao [9] we introduce the following boundary conditions

$$
w(0)=0, \quad E I \frac{d^{2} w(0)}{d x^{2}}=c \frac{d w(0)}{d x}, \quad w(L)=0, \quad E I \frac{d^{2} w(L)}{d x}=-d \frac{d w(L)}{d x}
$$

It is instructive to introduce the non-dimensional axial coordinate $\xi$, as well as the non dimensional rotational spring coefficients denoted as $C$ and $D$ respectively, respectively, as follows:

$$
\begin{aligned}
& \xi=\frac{x}{L} \\
& C=\frac{c L}{E I} \\
& D=\frac{d L}{E I}
\end{aligned}
$$

Equation (1) takes the form

$$
E I \frac{d^{4} w}{d^{4} \xi}=-q L^{4}
$$

whereas the boundary conditions read

$$
w(0)=0, \quad \frac{d^{2} w(0)}{d \xi^{2}}=C \frac{d w(0)}{d \xi}, \quad w(1)=0, \quad \frac{d^{2} w(1)}{d \xi}=-D \frac{d w(1)}{d \xi}
$$

Since Eq. (1) is a fourth order differential equation, with the displacement $w(\xi)$ constituting a fourth order polynomial as follows

$$
w(\xi)=-\frac{q L^{4}}{24 E I} \xi^{4}+\frac{1}{6} A_{1} \xi^{3}+\frac{1}{2} A_{2} \xi^{2}+A_{3} \xi+A_{4}
$$

where $A_{j}$ are constants. We consider $C \neq 0$ and $D \neq 0$. Satisfaction of boundary conditions Eq. (7) yields

$$
\begin{aligned}
w(\xi)= & -\frac{1}{24} \frac{q L^{4} \xi^{4}}{E I}+\frac{1}{12} \frac{q L^{4}(3 D+12+5 C+C D) \xi^{3}}{E I(4 D+12+4 C+C D)}-\frac{1}{24} \frac{q L^{4} C(D+6) \xi^{2}}{E I(4 D+12+4 C+C D)} \\
& -\frac{1}{12} \frac{q L^{4}(D+6) \xi}{E I(4 D+12+4 C+C D)}
\end{aligned}
$$

For $\xi=1 / 2$ we get the mid-span displacement

$$
w\left(\frac{1}{2}\right)=-\frac{1}{384} \frac{q L^{4}}{E I}+\frac{1}{96} \frac{q L^{4}(3 D+12+5 C+C D)}{E I(4 D+12+4 C+C D)}-\frac{1}{96} \frac{q L^{4} C(D+6)}{E I(4 D+12+4 C+C D)}
$$




$$
-\frac{1}{24} \frac{q L^{4}(D+6)}{E I(4 D+12+4 C+C D)}
$$

Consider several particular cases. If $C=0$ and $D=0$, the boundary conditions become

$$
w(0)=0, \quad \frac{d^{2} w(0)}{d \xi^{2}}=0, \quad w(1)=0, \quad \frac{d^{2} w(1)}{d \xi}=0
$$

Thus, the case $C=0, D=0$ corresponds to the beam that is simply supported at its both ends.

Equation (9) reduces to

$$
w(\xi)=-\frac{1}{24} \frac{q L^{4} \xi^{4}}{E I}+\frac{1}{12} \frac{q L^{4} \xi^{3}}{E I}-\frac{1}{24} \frac{q L^{4} \xi}{E I}
$$

and for $\xi=1 / 2$ we get a familiar expression

$$
w\left(\frac{1}{2}\right)=-\frac{5}{384} \frac{q L^{4}}{E I}
$$

In particular case $C \rightarrow \infty$ and $D \rightarrow \infty$, the boundary conditions Eq. (7) become

$$
w(0)=0, \quad \frac{d w(0)}{d \xi}=0, \quad w(1)=0, \quad \frac{d w(1)}{d \xi}=0
$$

Thus, for this case we have a beam that is clamped at both ends. Then solving Eq. (8) gives

$$
w(\xi)=-\frac{1}{24} \frac{q L^{4} \xi^{4}}{E I}+\frac{1}{12} \frac{q L^{4} \xi^{3}}{E I}-\frac{1}{24} \frac{q L^{4} \xi^{2}}{E I}
$$

Evaluating the displacement at $\xi=1 / 2$ we get

$$
w\left(\frac{1}{2}\right)=-\frac{1}{384} \frac{q L^{4}}{E I}
$$

In particular case $C=0$ and $D \rightarrow \infty$, the boundary conditions read

$$
w(0)=0, \quad \frac{d^{2} w(0)}{d \xi^{2}}=0, \quad w(1)=0, \quad \frac{d w(1)}{d \xi}=0
$$

Thus, for this case we have a simply supported beam at the left end and clamped at the right end. Solving Eq. (8) yields

$$
w(\xi)=-\frac{1}{24} \frac{q L^{4} \xi^{4}}{E I}+\frac{1}{16} \frac{q L^{4} \xi^{3}}{E I}-\frac{1}{48} \frac{q L^{4} \xi}{E I},
$$

and at $\xi=1 / 2$

$$
w\left(\frac{1}{2}\right)=-\frac{1}{192} \frac{q L^{4}}{E I}
$$

We consider the particular case $C=0$ and $D \neq 0$. The boundary conditions become

$$
w(0)=0, \quad \frac{d^{2} w(0)}{d \xi^{2}}=0, \quad w(1)=0, \quad \frac{d^{2} w(1)}{d \xi^{2}}=-D \frac{d w(1)}{d \xi}
$$

Thus, we are in the case of a simply supported beam at the left end and with a rotational spring and the pinned end at $x=L$. Equation (8) yields

$$
w(\xi)=-\frac{1}{24} \frac{q L^{4} \xi^{4}}{E I}+\frac{1}{16} \frac{q L^{4}(D+4) \xi^{3}}{E I(D+3)}-\frac{1}{48} \frac{q L^{4}(D+6) \xi}{E I(D+3)}
$$

Evaluating the displacement at the middle of the beam yields

$$
w\left(\frac{1}{2}\right)=-\frac{1}{384} \frac{q L^{4}}{E I}+\frac{1}{128} \frac{q L^{4}(D+4)}{E I(D+3)}-\frac{1}{96} \frac{q L^{4}(D+6)}{E I(D+3)}
$$




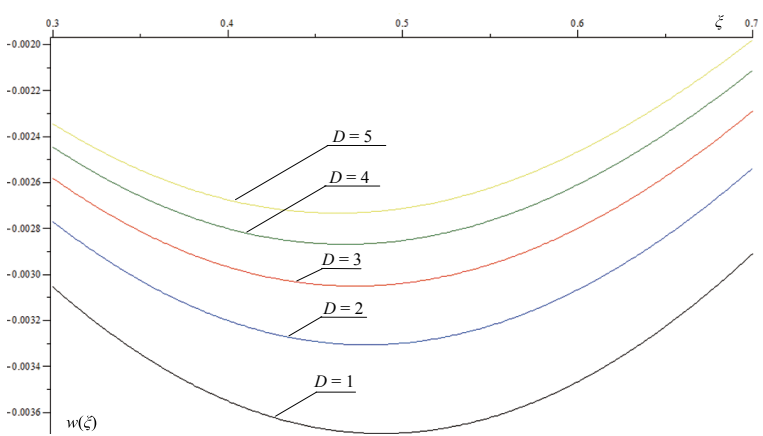

Fig. 2. Variation of the displacement $w(\xi)$ with $\xi$ for different values of the torsional rigidity $D$ of the right spring, for the vanishing left spring's rigidity $(C=0)$.

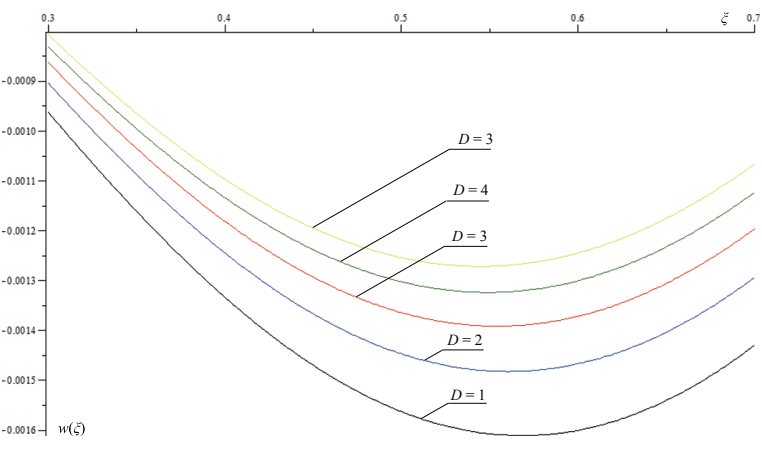

Fig. 3. Plot of the displacement $w(\xi)$ for different values of the right support's torsional rigidity $D$, for unbounded torsional rigidity of left support $(C=\infty)$.

In the particular case $C \rightarrow \infty$ and $D \neq 0$, the boundary conditions read

$$
w(0)=0, \quad \frac{d w(0)}{d \xi}=0, \quad w(1)=0, \quad \frac{d^{2} w(1)}{d \xi^{2}}=-D \frac{d w(1)}{d \xi}
$$

Thus, we are in the case of a clamped beam at the left end and with a rotational spring and the pinned end at $x=L$. Solving Eq. (8) using these boundary conditions yields

$$
w(\xi)=-\frac{1}{24} \frac{q L^{4} \xi^{4}}{E I}+\frac{1}{12} \frac{q L^{4}(D+5) \xi^{3}}{E I(D+4)}-\frac{1}{24} \frac{q L^{4}(D+6) \xi^{2}}{E I(D+4)}
$$

For $\xi=1 / 2$ we obtain

$$
w\left(\frac{1}{2}\right)=-\frac{1}{384} \frac{q L^{4}}{E I}+\frac{1}{96} \frac{q L^{4}(5+D)}{E I(D+4)}-\frac{1}{96} \frac{q L^{4}(D+6)}{E I(D+4)}
$$

Figure 2 depicts the variation of the displacement $w(\xi)$ with $\xi$ for different values of the torsional rigidity $D$ on the right spring, for the vanishing left spring's rigidity $(C=0)$. Figure 3 portrays the analogues dependence for various values for the right spring's rigidity $D$, for the particular case when $C$ is un-bounded.

\section{Application of classical interval analysis}

Now we resort to treating coefficients $C$ and $D$ as belonging to intervals, thus modeling their uncertainty.

Consider first the Eq. (21) that pertains to the beam with $C=0$, and $D \neq 0$. Let the right spring constant $D$ be an uncertain parameter, that varies in the interval $[\underline{D}, \bar{D}]$, where $\underline{D}$ is the lower possible value, whereas $\bar{D}$ is an upper possible value. Equation (21) contains the ratio $(D+4) /(D+3)$. According to the classical interval analysis it is evaluated as follows

$$
\frac{D+4}{D+3}=\frac{[\underline{D}+4, \bar{D}+4]}{[\underline{D}+3, \bar{D}+3]}=\left[\frac{\underline{D}+4}{\bar{D}+3}, \frac{\bar{D}+4}{\underline{D}+3}\right]
$$

For example, for $\underline{D}=0.95, \bar{D}=1.05$, this ratio becomes

$$
\frac{D+4}{D+3}=\left[\frac{0.95+4}{1.05+3}, \frac{1.05+4}{0.95+3}\right]=[1.222222222,1.278481013]
$$

On the other hand, one observes that

$$
\frac{D+4}{D+3}=1+\frac{1}{D+3}
$$


The interval evaluation of this expression results in

$$
1+\frac{1}{D+3}=1+\frac{1}{[\underline{D}+3, \bar{D}+3]}=1+\left[\frac{1}{\bar{D}+3}, \frac{1}{\underline{D}+3}\right]=\left[1+\frac{1}{\bar{D}+3}, 1+\frac{1}{\underline{D}+3}\right]
$$

For the considered numerical example, we get

$$
1+\frac{1}{3+[0.95,1.05]}=[1.246913580,1.253164557]
$$

which is not coincident with the result obtained in Eq. (27). We see that Eq. (27) leads to overestimation. For $\underline{D}=0.9, \bar{D}=1.1$, evaluation of the left side of Eq. (27) yields the interval $[1.195121951,1.307692308]$ whereas Eq. (29) results in the interval [1.243902439, 1.256410256].

As is seen in both cases the interval evaluated via Eq. (29) is included in the interval stemming from Eq. (27).

Likewise, for the ratio $(D+6) /(D+3)$ in Eq. (22) for $\underline{D}=0.95, \bar{D}=1.05$, we get $[1.716049383,1.784810127]$ via classical interval analysis, whereas if the ratio is simplified to $1+3 /(D+3)$ we obtain $[1.740740741$, $1.759493671]$. The evaluation for $\underline{D}=0.9, \bar{D}=1.1$ results in [1.682926829,1.820512821] via classical interval analysis, and $[1.731707317,1.769230769]$ using the simplified form for the ratio.

As is seen, if one takes care to have the same variable (in our case $D$ ) appear only once in the expression, then the resulting interval is sharper.

However, it is not always possible to realize this recommendation, since the evaluation procedure may be not analytical but purely numerical. These and other considerations lead to the idea that some radically different approach should be performed. Such a procedure is exposed in the following section.

\section{Deflection of the beam with torsional rigidities $C$ and $D$ treated as interval parameters}

Let $C$ and $D$ be interval variables such that they respectively take values from the following intervals

$$
C=[\underline{C}, \bar{C}], \quad D=[\underline{D}, \bar{D}]
$$

where $\underline{C}$ and $\underline{D}$ are lower bounds of the intervals $C$ and $D$, respectively; $\bar{C}$ and $\bar{D}$ are upper bounds of these respective intervals.

We introduce average values as follows

$$
\begin{aligned}
& C_{\text {ave }}=\frac{1}{2}(\underline{C}+\bar{C}) \\
& D_{\text {ave }}=\frac{1}{2}(\underline{D}+\bar{D})
\end{aligned}
$$

and deviations

$$
\begin{aligned}
& C_{\text {dev }}=\frac{1}{2}(\bar{C}-\underline{C}) \\
& D_{\text {dev }}=\frac{1}{2}(\bar{D}-\underline{D})
\end{aligned}
$$

In these circumstances

$$
\begin{aligned}
& \underline{C}=C_{a v e}-C_{d e v}, \quad \bar{C}=C_{a v e}+C_{d e v} \\
& \underline{D}=D_{a v e}-D_{d e v}, \quad \bar{D}=D_{a v e}+D_{d e v}
\end{aligned}
$$


Since upper and lower bounds of intervals are symmetrically placed with respect to the average values, it is instructive to represent all points of the interval using some trigonometric function, as follows

$$
\begin{aligned}
& C=C_{\text {ave }}+C_{\text {dev }} \sin (t) \\
& D=D_{\text {ave }}+D_{\text {dev }} \sin (t)
\end{aligned}
$$

where $t$ is an artificial parameter that varies in the interval $[-\pi / 2, \pi / 2]$. The value $t=-\pi / 2$ then corresponds to the lower bound, whereas $t=\pi / 2$ is associated with the upper bound. In these circumstances the static deflection becomes a function of both axial coordinate $\xi$ and artificial coefficient $t$.

It should be noted that the choice made in Eqs (38) and (39) of the trigonometric function is not absolutely necessary. Other functions may be equally applicable. It also should be stressed that the by-product of such a choice signifies that the stiffness quantities at both ends are somehow coupled. Naturally, one can inquire if such a coupling phenomenon can be justified. One must note that when interval analysis problem is formulated, no information is provided on dependency between two intervals. Hence it appears legitimate to introduce any assumption that may provide sharper bounds than obtainable otherwise. For example, dependency, or coupling may be justified if the springs are produced in the same company, using the same manufacturing process. If however the information is communicated that the springs come from different sources, and are manufactured by differing processes, the coupling assumption cannot be validated.

Since the spring constant are considered as interval variables, the deflection at each cross section becomes likewise an interval variable.

We are interested in finding the lower and upper bounds of displacement at each $\xi$.

There is a possibility of using rules of interval mathematics to achieve our goal. However, it is well known that interval mathematics may yield considerable, and often times, a dramatic overestimation.

In these circumstances we utilize numerical technique to obtain the extrema of the displacement. To this end, for every value of $\xi$, we utilize MATLAB command of minimization and maximization with respect to the parameter $t$ in the interval $[-\pi / 2, \pi / 2]$.

Consider now the expression Eq. (26) in order to check how the proposed parameterization in Eqs (38) and (39) performs. Using representation given in Eqs (38) and (39), Eq. (26) becomes:

$$
\frac{D+4}{D+3}=\frac{D_{a v e}+D_{\text {dev }} \sin (t)+4}{D_{\text {ave }}+D_{\text {dev }} \sin (t)+3}
$$

For the specific case $D_{\text {ave }}=1$ and $D_{\text {dev }}=0.05$, (i.e. for $\underline{D}=0.95$ and $\bar{D}=1.05$ ), the ratio becomes

$$
\begin{aligned}
\frac{D_{a v e}+D_{d e v} \sin (t)+4}{D_{a v e}+D_{d e v} \sin (t)+3} & =\left[\frac{1+0.05 \sin \left(\frac{\pi}{2}\right)+4}{1+0.05 \sin \left(\frac{\pi}{2}\right)+3}, \frac{1+0.05 \sin \left(-\frac{\pi}{2}\right)+4}{1+0.05 \sin \left(-\frac{\pi}{2}\right)+3}\right] \\
& =[1.246913580,1.253164557]
\end{aligned}
$$

which coincides with exact interval given in Eq. (30). One has to note that using the command MAXIMIZE in MAPLE $^{\circledR}$, yields the same interval. For another case $D_{\text {ave }}=1$ and $D_{\text {dev }}=0.1$, (i.e. for $\underline{D}=0.9$ and $\bar{D}=1.1$ ), it becomes

$$
\begin{aligned}
\frac{D_{\text {ave }}+D_{\text {dev }} \sin (t)+4}{D_{\text {ave }}+D_{\text {dev }} \sin (t)+3} & =\left[\frac{1+0.1 \sin \left(\frac{\pi}{2}\right)+4}{1+0.1 \sin \left(\frac{\pi}{2}\right)+3}, \frac{1+0.1 \sin \left(-\frac{\pi}{2}\right)+4}{1+0.1 \sin \left(-\frac{\pi}{2}\right)+3}\right] \\
& =[1.243902439,1.256410256]
\end{aligned}
$$

which coincides with exact interval listed in Section 3.

As is seen the representation given in Eq. (28) is avoided by parameterization of the intervals.

Figure 4 is a plot of the displacement $w$ as a function of $\xi$ and $t$, for data following values are taken: $q=$ $10 \mathrm{~N} / \mathrm{m}, L=1 \mathrm{~m}, E=210 \mathrm{GPa}, I=1.067 \cdot 10^{-3} \mathrm{~m}^{4}, C_{a v e}=0.5 \mathrm{~N} \cdot \mathrm{m} \cdot \mathrm{rad}^{-1}, C_{\text {dev }}=1 \mathrm{~N} \cdot \mathrm{m} \cdot \mathrm{rad}^{-1}, D_{a v e}=$ $0.5 \mathrm{~N} \cdot \mathrm{m} \cdot \mathrm{rad}^{-1}, D_{\text {dev }}=1 \mathrm{~N} \cdot \mathrm{m} \cdot \mathrm{rad}^{-1}$.

As seen on Fig. 4, the minima and the maxima of the deflection are obtained for $t=\pi / 2$ and $t=-\pi / 2$, respectively, due to the fact that $w(\xi, t)$ is a negative quantity. 


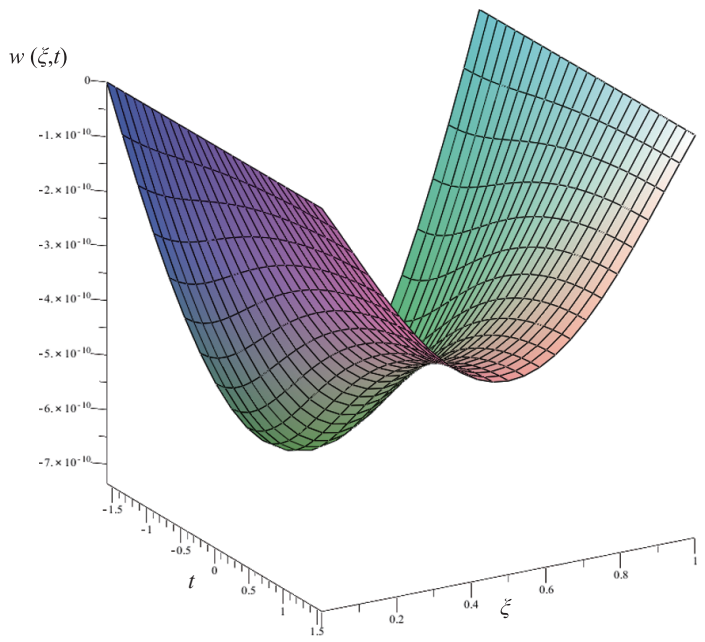

Fig. 4. Deflection $w$ of the beam as a function of the parameter $t$ and the axial coordinate $\xi$.

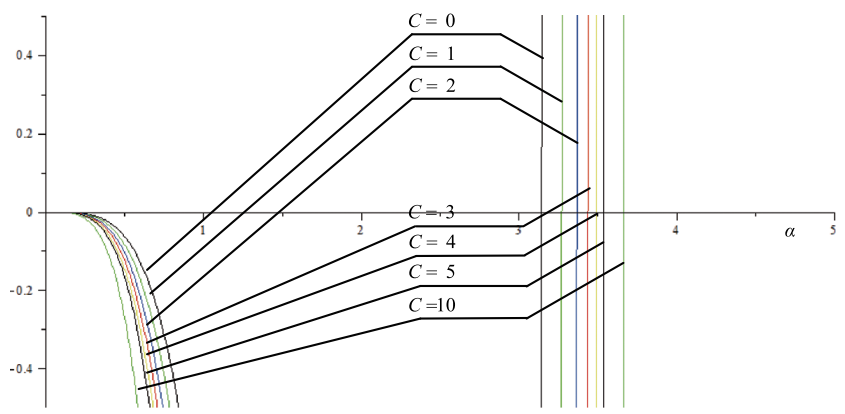

Fig. 5. Plot of $f$ as a function of $\alpha$ in the case of a beam with a rotational spring at its left end and simply supported at its right end.

\section{Natural frequencies}

The governing differential Eq. for vibrations of uniform beams reads

$$
E I \frac{d^{4} W}{d x^{4}}-\rho A \omega^{2} W=0
$$

We introduce the non-dimensional parameter $\xi$ as for Eq. (6). Equation (43) becomes

$$
E I \frac{d^{4} w}{d \xi^{4}}-\rho L^{4} A \omega^{2} w=0
$$

We denote the parameter $\alpha$ defined as follows

$$
\alpha^{4}=\frac{\rho A L^{4} \omega^{2}}{E I}
$$

Thus, Eq. (43) now reads

$$
\frac{d^{4} W}{d \xi^{4}}-\alpha^{4} W=0
$$

The displacement $w(\xi)$ can be written as follows

$$
W(\xi)=B_{1} K_{1}(\alpha \xi)+B_{2} K_{2}(\alpha \xi)+B_{3} K_{3}(\alpha \xi)+B_{4} K_{4}(\alpha \xi)
$$

where $K_{j}(x)$ are Krylov functions defined as follows

$$
\begin{array}{ll}
K_{1}=\frac{1}{2}[\cosh (x)+\cos (x)] & K_{3}=\frac{1}{2}[\cosh (x)-\cos (x)] \\
K_{2}=\frac{1}{2}[\sinh (x)+\sin (x)] & K_{4}=\frac{1}{2}[\sinh (x)-\sin (x)]
\end{array}
$$

Boundary conditions at $\xi=0$ in Eq. (7) yield

$$
B_{1}=0
$$


Table 1

First ten frequencies of a simply supported beam at its right end and with a rotational spring at its left end obtained for various values of the torsional rigidity $(D=0)$

\begin{tabular}{|c|c|c|c|c|c|c|c|}
\hline & $C=0$ & $C=1$ & $C=2$ & $C=3$ & $C=4$ & $C=5$ & $C=5$ \\
\hline$\alpha_{S p r i n g}-S_{1}$ & 3.1415927 & 3.2732861 & 3.3666028 & 3.4364157 & 3.4906904 & 3.5341247 & 3.6646442 \\
\hline$\alpha_{S p r i n g}-S_{2}$ & 6.2831853 & 6.3559854 & 6.4171989 & 6.4692323 & 6.5139114 & 6.5526320 & 6.6874331 \\
\hline$\alpha_{S p r i n g}-S_{3}$ & 9.4247780 & 9.4748620 & 9.5195542 & 9.5595843 & 9.5955770 & 9.6280641 & 9.7515722 \\
\hline$\alpha_{S p r i n g}-S_{4}$ & 12.5663706 & 12.6045070 & 12.6395558 & 12.6718219 & 12.7015804 & 12.7290790 & 12.8396341 \\
\hline$\alpha_{S p r i n g}-S_{5}$ & 15.7079633 & 15.7387441 & 15.7675318 & 15.7944796 & 15.8197307 & 15.8434174 & 15.9423269 \\
\hline$\alpha_{S p r i n g}-S_{6}$ & 18.8495560 & 18.8753562 & 18.8997655 & 18.9228713 & 18.9447566 & 18.9654995 & 19.0544989 \\
\hline$\alpha_{S p r i n g}-S_{7}$ & 21.9911486 & 22.0133540 & 22.0345344 & 22.0547441 & 22.0740353 & 22.0924577 & 22.1731187 \\
\hline$\alpha_{\text {Spring }}-S_{8}$ & 25.1327412 & 25.1522302 & 25.1709330 & 25.1888853 & 25.2061221 & 25.2226768 & 25.2963035 \\
\hline$\alpha_{S p r i n g}-S_{9}$ & 28.2743339 & 28.2916983 & 28.3084405 & 28.3245856 & 28.3401579 & 28.3551810 & 28.4228310 \\
\hline$\alpha_{\text {Spring }}-S_{10}$ & 31.4159265 & 31.4315837 & 31.4467365 & 31.4614029 & 31.4756006 & 31.48934680 & 31.5518747 \\
\hline
\end{tabular}

$$
B_{3}=\frac{C}{\alpha} B_{2}
$$

Thus we get

$$
W(\xi)=B_{2}\left[K_{2}(\alpha \xi)+\frac{C}{\alpha} K_{3}(\alpha \xi)\right]+B_{4} K_{4}(\alpha \xi)
$$

Boundary conditions at $\xi=1$ in Eq. (7) yield

$$
\begin{aligned}
& B_{2}\left[K_{2}(\alpha)+\frac{C}{\alpha} K_{3}(\alpha)\right]+B_{4} K_{4}(\alpha)=0 \\
& B_{2}\left[D\left(\alpha K_{1}(\alpha)+C K_{2}(\alpha)\right)+\left(\alpha^{2} K_{4}(\alpha)+\alpha C K_{1}(\alpha)\right)\right]+B_{4}\left[\alpha^{2} K_{2}(\alpha)+\alpha D K_{3}(\alpha)\right]=0
\end{aligned}
$$

We introduce the function $f$, defined as the determinant of Eqs (52) and (53), as a function of $\alpha$ and we get

$$
\begin{aligned}
f(\alpha)= & -2 \sinh (\alpha) \sin (\alpha) \alpha^{2}+D \alpha \sinh (\alpha) \cos (\alpha)+C \alpha \cos (\alpha) \sinh (\alpha)-D \alpha \sin (\alpha) \cosh (\alpha) \\
& -C \alpha \cosh (\alpha) \sin (\alpha)+C D \cosh (\alpha) \cos (\alpha)-C D
\end{aligned}
$$

We consider several particular cases. We first treat the case $D=0$. Thus Eq. (54) becomes

$$
f(\alpha)=-2 \sinh (\alpha) \sin (\alpha) \alpha^{2}+C \alpha \cos (\alpha) \sinh (\alpha)-C \alpha \cosh (\alpha) \sin (\alpha)
$$

We would like to consider several sub-cases.

In the first particular case we set $C=0$, in addition to $D=0$. This means that we are in the case of a simply supported beam at both ends. Then Eq. (55) is highly simplified and reads

$$
f(\alpha)=-2 \sinh (\alpha) \sin (\alpha) \alpha^{2}
$$

The first ten frequencies for this beams are, respectively, $\alpha_{S-S_{1}}=3.141592654, \alpha_{S-S_{2}}=6.283185308$, $\alpha_{S-S_{3}}=9.424777961, \alpha_{S-S_{4}}=12.56637061, \alpha_{S-S_{5}}=15.70796327, \alpha_{S-S_{6}}=18.84955592, \alpha_{S-S_{7}}=$ $21.99114858, \alpha_{S-S_{8}}=25.13274123, \alpha_{S-S_{9}}=28.27433388$ and $\alpha_{S-S_{10}}=31.41592654$. These numerical values obtained by MAPLE ${ }^{\circledR}$, are in accordance with the exact formula $\alpha=k \pi, k=1,2, \ldots$, as they should be.

In this second particular case, we consider the limiting case $C \rightarrow \infty$ in addition to $D=0$, which corresponds to beam that is clamped at one end and simply supported at the other. The determinant reads

$$
f(\alpha)=\frac{1}{2} \cosh (\alpha) \sin (\alpha)-\frac{1}{2} \cos (\alpha) \sinh (\alpha)
$$

The first ten frequencies for this beam are, respectively, $\alpha_{C-S_{1}}=3.926602312, \alpha_{C-S_{2}}=7.068582745$, $\alpha_{C-S_{3}}=10.21017612, \alpha_{C-S_{4}}=13.35176878, \alpha_{C-S_{5}}=16.49336143, \alpha_{C-S_{6}}=19.63495408, \alpha_{C-S_{7}}=$ $22.77654674, \alpha_{C-S_{8}}=25.91813939, \alpha_{C-S_{9}}=29.05973204$ and $\alpha_{C-S_{10}}=32.20132470$. 
Table 2

First ten frequencies of a beam with a rotational spring at both ends obtained for various values of the torsional rigidity

\begin{tabular}{lrrrrrrr}
\hline & \multicolumn{1}{c}{$C=0$} & \multicolumn{1}{c}{$C=1$} & \multicolumn{1}{c}{$C=2$} & \multicolumn{1}{c}{$C=3$} & \multicolumn{1}{c}{$C=4$} & \multicolumn{1}{c}{$C=5$} & \multicolumn{1}{c}{$C=10$} \\
\hline$\alpha_{\text {Spring-Spring }_{1}}$ & 3.1415927 & 3.3987993 & 3.5768301 & 3.7096901 & 3.8135395 & 3.8973550 & 4.1556642 \\
$\alpha_{\text {Spring-Spring }}$ & 6.2831853 & 6.4272591 & 6.5465721 & 6.6471633 & 6.7332056 & 6.8076915 & 7.0682493 \\
$\alpha_{\text {Spring-Spring }}$ & 9.4247780 & 9.5244522 & 9.6126793 & 9.6912605 & 9.7616506 & 9.8250346 & 10.0656791 \\
$\alpha_{\text {Spring-Spring }}$ & 12.5663706 & 12.6424234 & 12.7119707 & 12.7757489 & 12.8343979 & 12.8884748 & 13.1052639 \\
$\alpha_{\text {Spring-Spring }}$ & 15.7079633 & 15.7694090 & 15.8266825 & 15.8801473 & 15.9301330 & 15.9769375 & 16.1717915 \\
$\alpha_{\text {Spring-Spring }}$ & 18.8495560 & 18.9010880 & 18.9497241 & 18.9956678 & 19.0391085 & 19.0802214 & 19.2561282 \\
$\alpha_{\text {Spring-Spring }}$ & 21.9911486 & 22.0355157 & 22.0777579 & 22.1180000 & 22.1563596 & 22.1929479 & 22.3527502 \\
$\alpha_{\text {Spring-Spring }}$ & 25.1327412 & 25.1716897 & 25.2090139 & 25.2447952 & 25.2791117 & 25.3120376 & 25.4581581 \\
$\alpha_{\text {Spring-Spring }}$ & 28.2743339 & 28.3090417 & 28.3424681 & 28.3746692 & 28.4056989 & 28.4356095 & 28.5700446 \\
$\alpha_{\text {Spring-Spring }}$ & 31.4159265 & 31.4472256 & 31.4774882 & 31.5067545 & 31.5350636 & 31.5624530 & 31.6868348 \\
\hline
\end{tabular}

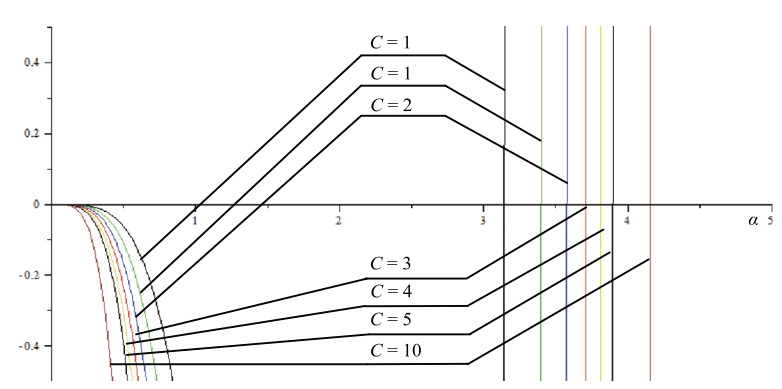

Fig. 6. Plot of $f$ as a function of $\alpha$ in the case of a beam with two rotational springs at its ends.

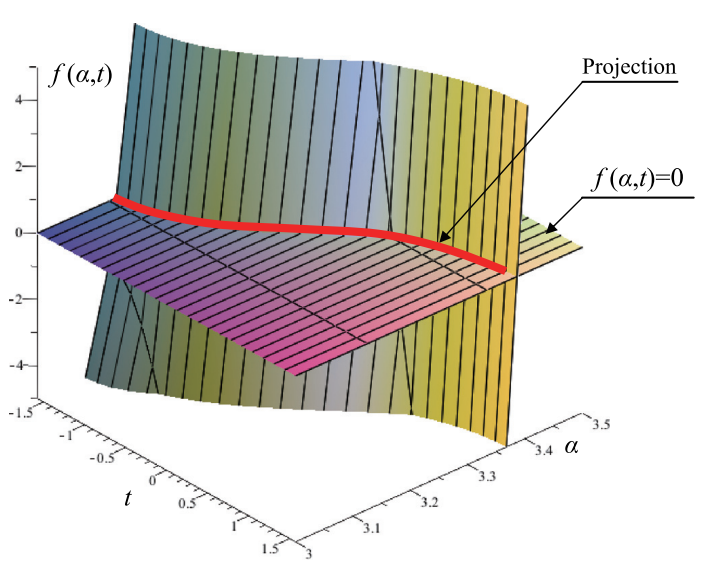

Fig. 7. Intersection of the three-dimensional plot of function $f(\alpha, t)$ with the plane $f(\alpha, t)=0$.

Consider the more general case $C \neq 0, D \neq 0$. We plot of $f$ as a function of $\alpha$ for $\alpha_{S-S} \leqslant \alpha \leqslant \alpha_{C-S}$ with parameter $C$ set at various values as depicted in Fig. 5. It shows function $f(\alpha)$ in a zoomed form. Left branch of $f(\alpha)$ is easily seen. The right branch is shown is the vicinity when $f(\alpha)$ becomes zero. Parts of the curves are not shown because of scaling reasons.

We determine the ten first frequencies for several specific values of $C$, which are listed in Table 1.

As a second particular case,we consider identical springs at the ends of the beam $(C=D)$. In this case, Eq. (54) reduces to

$$
f(\alpha)=-2 \sinh (\alpha) \sin (\alpha) \alpha^{2}+2 C \alpha[\sinh (\alpha) \cos (\alpha)-\cosh (\alpha) \sin (\alpha)]+C^{2} \cosh (\alpha) \cos (\alpha)-C^{2}
$$

Figure 6 is a plot of $f$ as a function of $\alpha$ with the parameter $C$ set for its various values The first ten frequencies for these cases as listed in Table 2.

Now we turn our attention to the realistic case when spring constants constitute interval parameters.

\section{Natural frequencies with torsonal rigidities treated as interval parameters}

We substitute Eqs (38) and (39) into Eq. (54) and to get

$$
\begin{aligned}
f(\alpha, t)= & -2 \sinh (\alpha) \sin (\alpha) \alpha^{2}+\left[D_{\text {ave }}+D_{\text {dev }} \sin (t)\right] \alpha \sinh (\alpha) \cos (\alpha) \\
& +\left[C_{\text {ave }}+C_{\text {dev }} \sin (t)\right] \alpha \cos (\alpha) \sinh (\alpha)-\left[D_{\text {ave }}+D_{\text {dev }} \sin (t)\right] \alpha \sin (\alpha) \cosh (\alpha) \\
& -\left[C_{\text {ave }}+C_{\text {dev }} \sin (t)\right] \alpha \cosh (\alpha) \sin (\alpha)
\end{aligned}
$$




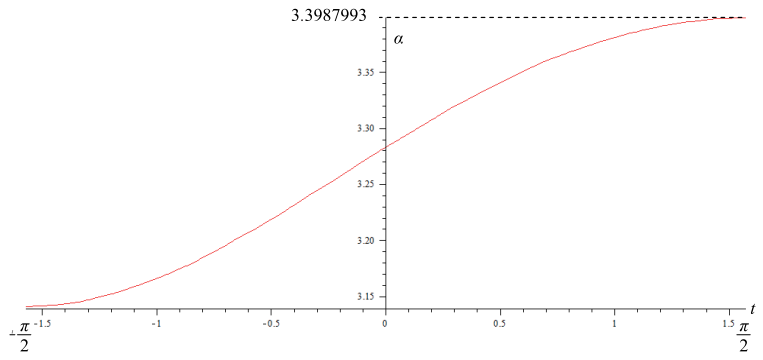

Fig. 8. Variation of the first natural frequency with respect to the parameter $t$.

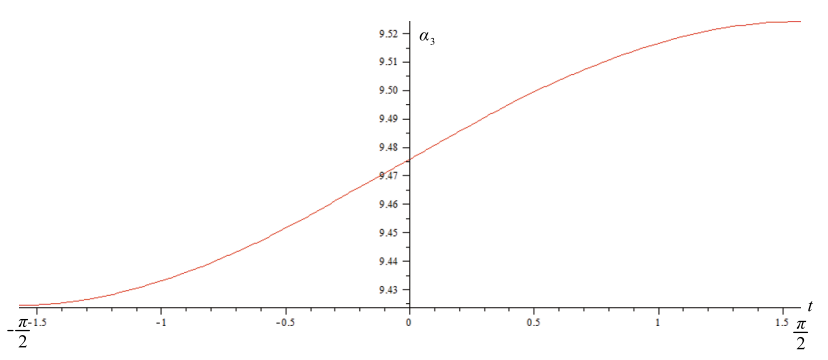

Fig. 10. Variation of the third natural frequency parameter with respect to the parameter $t$.

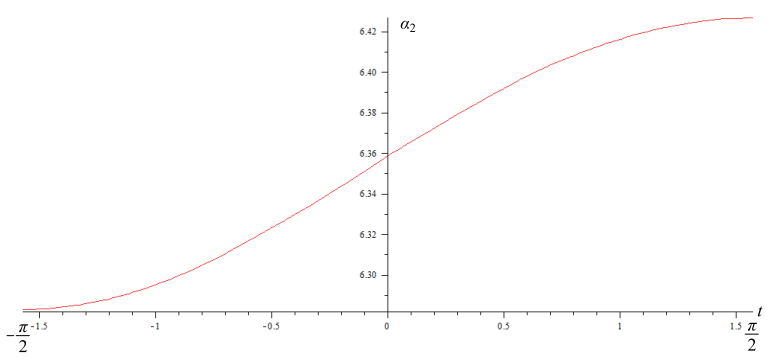

Fig. 9. Variation of the second natural frequency parameter with respect to the parameter $t$.

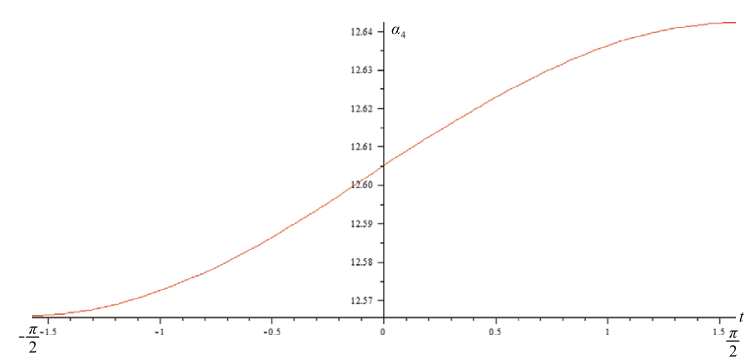

Fig. 11. Variation of the fourth natural frequency parameter with respect to the parameter $t$.

$$
\begin{aligned}
& +\left[C_{\text {ave }}+C_{\text {dev }} \sin (t)\right]\left[D_{\text {ave }}+D_{\text {dev }} \sin (t)\right] \cosh (\alpha) \cos (\alpha) \\
& -\left[C_{\text {ave }}+C_{\text {dev }} \sin (t)\right]\left[D_{\text {ave }}+D_{\text {dev }} \sin (t)\right]
\end{aligned}
$$

Numerical analysis is performed as follows. A three-dimensional plot of $f(\alpha, t)$ according to Eq. (59) is plotted in a region $[-\pi / 2 \leqslant t \leqslant \pi / 2,0 \leqslant \alpha \leqslant 5]$ for the first natural frequency. The projection of this curve on plane $f(\alpha, t)=0$ is shown in Fig. 7 by solid line.

The natural frequencies are obtained solving Eq. (59) for each value of $t$. We use following values for the interval parameters $\underline{C}=\underline{D}=0, \bar{C}=\bar{D}=1$. These values correspond to $C_{\text {ave }}=D_{\text {ave }}=0.5$ and $C_{\text {dev }}=D_{\text {dev }}=0.5$, we obtain the variation of the first natural frequency as a function of parameter $t$ depicted in Fig. 8. The lowest possible value of the parameter $\alpha$ is achieved for $t=-\pi / 2$ corresponding to lowest possible values $\underline{C}=\underline{D}=0$. The minimal value equals $\alpha=\pi$ corresponding to simply supported beam. Greatest value of $\alpha$ equals $\alpha_{\max }=$ 3.2732861 which is close agreement with value in Table 2 for $C=D=1$.

We also get the curve for the following higher frequencies as depicted in Figs 9-12.

The results of evaluation of the intervals $[\underline{\alpha}, \alpha]$ for the natural frequency parameters $\alpha_{n}(n=1-10)$ are listed in Table 3.

\section{Natural frequencies of beams with uncertain mass density}

Let us now consider possible complicating effect of the uncertain mass density $\rho$ treated as an interval parameter too $\rho=[\underline{\rho}, \bar{\rho}]$. Then we introduce average mass density $\rho_{\text {ave }}$ and deviation $\rho_{\text {dev }}$ as follows

$$
\begin{aligned}
& \rho_{\text {ave }}=\frac{\bar{\rho}+\underline{\rho}}{2}, \\
& \rho_{\text {dev }}=\frac{\bar{\rho}-\underline{\rho}}{2},
\end{aligned}
$$

and put $\rho(t)$ as the following function of the artificial parameter $t$ :

$$
\rho=\rho_{\text {ave }}+\rho_{\text {dev }} \sin (t)
$$


For simplicity, we will use a single term Galerkin approximation to deal with this hybrid case. We approximate the mode shape $w(\xi)$ as follows

$$
w(\xi)=A \psi(\xi)
$$

where $\psi(\xi)$ is a coordinate function that satisfies all boundary conditions. It is natural to take expression in Eq. (9) representing static solution as such an approximating function. Hence

$$
\psi(\xi)=\xi^{4}-\frac{2 \cdot(3 D+12+5 C+C D)}{(4 D+12+4 C+C D)} \xi^{3}+\frac{C(D+6)}{(4 D+12+4 C+C D)} \xi^{2}+\frac{2 \cdot(D+6)}{(4 D+12+4 C+C D)} \xi
$$

which is proportional to Eq. (9). Naturally $\psi(\xi)$, as a static solution, does not satisfy the differential Eq. (43) for vibrating beam. Rather, substitution of $\psi$ into Eq. (44) yields an error, denoted as $\varepsilon(\xi)$ :

$$
\varepsilon(\xi)=E I \frac{d^{4} \psi}{d \xi^{4}}-\rho L^{4} A \omega^{2} \psi
$$

Followingthe Bubnov-Galerkin method, we demand

$$
\int_{0}^{1} \varepsilon(\xi) \psi(\xi) d \xi=0
$$

Equation (65) yields

$$
\alpha^{4}=\frac{504[72+9 D+9 C+C D][C D+4 C+4 D+12]}{1140 C+1140 D+272 C D+76 C^{2}+C^{2} D^{2}+17 C^{2} D+17 C D^{2}+76 D^{2}+4464}
$$

Taking into account Eq. (45) and substituting Eqs (38), (39) and (60) in Eq. (66) results in

$$
\begin{aligned}
\omega^{2}=\frac{E I}{L^{4} \rho(t) A} \frac{504[72+9 D(t)+9 C(t)+C(t) D(t)][C(t) D(t)+4 C(t)+4 D(t)+12]}{1140 C(t)+1140 D(t)+272 C(t) D(t)+76[C(t)]^{2}+[C(t)]^{2}[D(t)]^{2}} \\
+17[C(t)]^{2} D(t)+17 C(t)[D(t)]^{2}+76[D(t)]^{2}+4464
\end{aligned}
$$

One can also use the classical Rayleigh-Ritz method requiring that the maximum energy of the beam

$$
U_{\max }=\frac{1}{2} \int_{0}^{L} E I\left(\frac{d^{2} \psi}{d x^{2}}\right)^{2} d x+\frac{1}{2} c^{2}\left(\frac{d \psi}{d x}\right)_{x=0}^{2}+\frac{1}{2} d\left(\frac{d \psi}{d x}\right)_{x=L}^{2}
$$

to equal its maximum kinetic energy

$$
T_{\max }=\frac{1}{2} \omega^{2} \int_{0}^{L} \rho A \psi^{2}(x) d x
$$

yielding the squared natural frequency as follows, after some algebra:

$$
\omega^{2}=\frac{E I}{\rho A L^{4}} \frac{\int_{0}^{1}\left[\psi^{\prime \prime}(\xi)\right]^{2} d \xi+C\left[\psi^{\prime}(\xi)\right]_{\xi=0}^{2}+D\left[\psi^{\prime}(\xi)\right]_{\xi=1}^{2}}{\int_{0}^{1}\left[\psi^{2}(\xi)\right] d \xi}
$$


Table 3

Lower and upper values of first ten frequencies of a beam with a rotational spring at both ends obtained for the torsional rigidity being interval parameter

\begin{tabular}{ccc}
\hline & $\underline{\alpha}$ & $\bar{\alpha}$ \\
\hline 1 & 3.141592654 & 3.398799335 \\
2 & 6.283185307 & 6.427259073 \\
3 & 9.424777961 & 9.524452159 \\
4 & 12.56637061 & 12.64242338 \\
5 & 15.70796327 & 15.76940898 \\
6 & 18.84955592 & 18.90108802 \\
7 & 21.99114858 & 22.03551570 \\
8 & 25.13274123 & 25.17168972 \\
9 & 28.27433388 & 28.30904172 \\
10 & 31.41592654 & 31.44722560 \\
\hline
\end{tabular}

Table 4

Evaluation of natural frequencies

\begin{tabular}{cccccc}
\hline$C$ & $D$ & Exact solution & Galerkin & Fung & Rayleigh-Ritz \\
\hline 0 & 0 & 3.14159265 & 3.14271518 & 3.14159265 & 3.14271518 \\
0.5 & 0 & 3.21362953 & 3.21490155 & 3.21517127 & 3.21490155 \\
5 & 5 & 3.89735503 & 3.90094146 & 3.97585715 & 3.90094146 \\
10 & 10 & 4.15566423 & 4.16066619 & 4.24308226 & 4.16066619 \\
50 & 50 & 4.56291875 & 4.57039751 & 4.59429164 & 4.57039751 \\
10 & 0 & 3.66464421 & 3.66764009 & 3.69233746 & 3.66764009 \\
50 & 0 & 3.85514477 & 3.85913766 & 3.86794215 & 3.85913766 \\
100 & 100 & 4.64131896 & 4.64915752 & 4.65148975 & 4.64915751 \\
$\infty$ & 0 & 3.92660231 & 3.93079017 & 3.92699082 & 3.93079017 \\
$\infty$ & $\infty$ & 4.73004074 & 4.73813722 & 4.71238898 & 4.73813722 \\
\hline
\end{tabular}

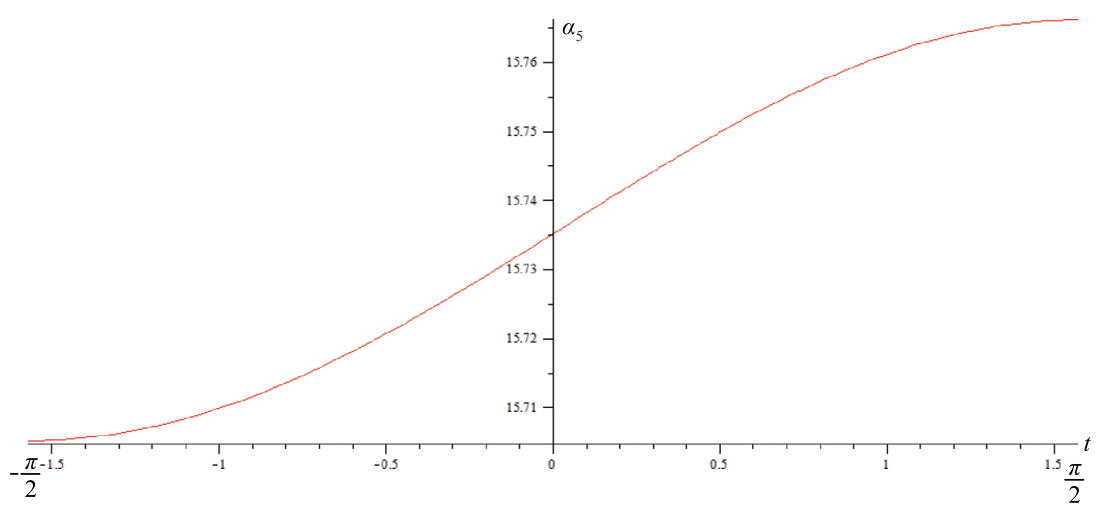

Fig. 12. Variation of the fifth natural frequency parameter with respect to the parameter $t$..

Table 3 lists exact and approximate natural frequencies, determined by both the Galerkin and the Rayleigh methods. As expected, the squared natural frequency in Eq. (70) coincides with Eq. (67) furnished by the BubnovGalerkin method.

It must be stressed that, numerical evaluation of the exact natural frequencies "could be clumsy", in terminology of Fung [10]. Therefore, one can resort additionally to their asymptotic evaluation. Noting that for high natural frequencies

$$
\sinh (\alpha) \approx \cosh (\alpha)
$$

Equation (66) can be divided by $\sinh (\alpha)$ to obtain, after some further simplifications the following formula

$$
\alpha_{n}=n \pi+\tan ^{-1}\left[\frac{(C+D) n \pi+C D}{2 n^{2} \pi^{2}+(C+D) n \pi}\right]
$$

derived by Fung [10], $n$ denoting the sequential member of the frequency parameter. Results of evaluation of natural frequencies based upon Fung's formula are also listed in Table 3. It should be noted that Fung's [10] approach is equivalent to application of Bolotin's dynamic edge effect method (see Elishakoff [11]).

Variation of $\omega$ with parameter $t$ is depicted in Fig. 13. The average value $\rho_{\text {ave }}$ is fixed at $7800 \mathrm{~kg} \cdot \mathrm{m}^{-3}$.

We observe an interesting phenomenon in Fig. 13, namely the behavior of the curves varies qualitatively for different values of $\rho_{\text {dev }}$. Specifically, the frequency is monotonically increasing with the parameter $t$ for $\rho_{d e v}=$ 1000 , whereas the frequency is monotonically decreasing with the parameter $t$ for $\rho_{d e v}=2000$. Remarkably, the behavior is not monotonic when $\rho=1270$. It should be noted that the lack of monotonicity shows that performing a simple (but very popular) vertex analysis in this case would yield erroneous results. 
Table 5

Natural frequency intervals

\begin{tabular}{cccc}
\hline$n$ & Methods & $\underline{\alpha}$ & $\bar{\alpha}$ \\
\hline 1 & Exact & 3.141592654 & 3.398799335 \\
& Bubnov-Galerkin & 3.142715180 & 3.400513100 \\
\multirow{2}{*}{2} & Fung & 3.141592654 & 3.414491419 \\
& Exact & 6.283185307 & 6.427259073 \\
3 & Fung & 6.283185306 & 6.430342534 \\
& Exact & 9.424777961 & 9.524452159 \\
4 & Fung & 9.424777959 & 9.525450768 \\
& Exact & 12.56637061 & 12.64242338 \\
5 & Fung & 12.56637062 & 12.64286564 \\
& Exact & 15.70796327 & 15.76940898 \\
6 & Fung & 15.70796327 & 15.76964178 \\
& Exact & 18.84955592 & 18.90108802 \\
7 & Fung & 18.84955592 & 18.90122519 \\
& Exact & 21.99114858 & 22.03551570 \\
8 & Fung & 21.99114858 & 22.03560319 \\
& Exact & 25.13274123 & 25.17168972 \\
9 & Fung & 25.13274124 & 25.17174890 \\
& Exact & 28.27433388 & 28.30904172 \\
\multirow{2}{*}{10} & Fung & 28.27433388 & 28.30908357 \\
& Exact & 31.41592654 & 31.44722560 \\
& Fung & 31.41592654 & 31.44725630 \\
\hline
\end{tabular}

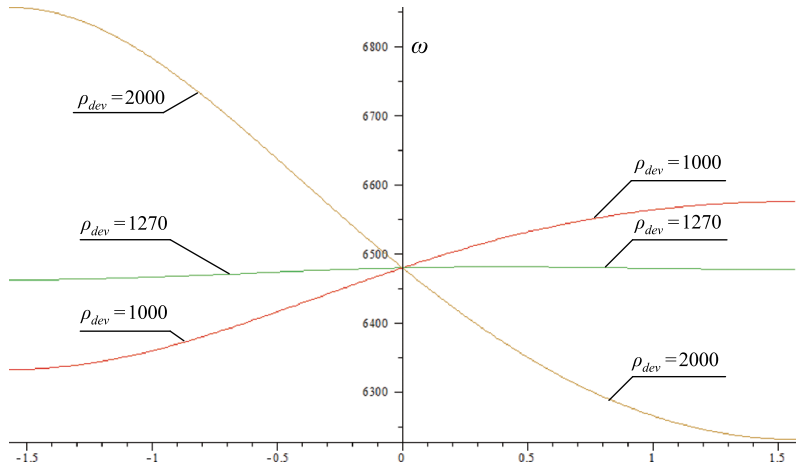

Fig. 13. Variation of the first natural frequency $\omega$ as a function of the parameter $t$ for various values for $\rho_{d e v}$.

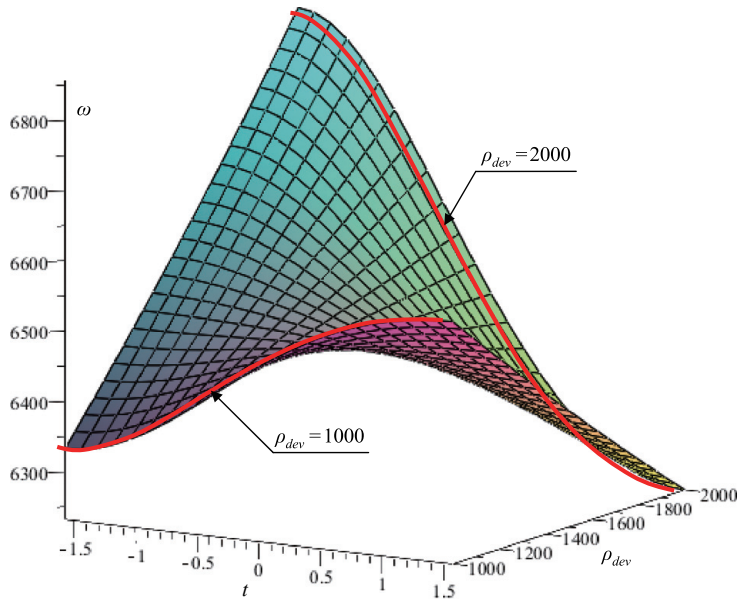

Fig. 14. Variation of the first natural frequency $\omega$ with respect to the parameter $t$ and the value of $\rho_{d e v}$.

It appears instructive to depict the variation of the natural frequency $\omega$ with respect to the two parameters: $t$ and $\rho_{\text {dev }}$ as shown in Fig. 14.

As is seen in Fig. 14 the qualitative and quantitative behavior of the curve $\omega$ versus artificial parameter $t$ is sensitive to value $\rho_{\text {dev }}$. This fact correlates with another observation of Fig. 13.

Natural frequency intervals yielded both by exact and approximate techniques are listed in Table 4 . For the fundamental natural frequency intervals obtained via exact solution as well as Bubnov-Galerkin method along with results associated with Fung's asymptotic formula are reported. For the other natural frequencies only exact solution and Fung's approximation are utilized. One cannot make a general conclusion as far which method should be preferred to obtain the smallest interval of natural frequency. As far the Bubnov-Galerkin method is concerned the lower bound yielded by it is greater than the lower bound produced by the exact solution; likewise the upper bound obtained via the Bubnov-Galerkin method turns out to be greater than its exact counterpart. Lower bounds yielded by the Fung's formula are nearly coincident with those furnished by the exact characteristic equation. The upper bound delivered by the Fung's formula turns out to be in excess of the upper bound produced by the exact solution. 
Still, the closeness of the exact and approximate solutions appears to be excellent.

\section{Conclusion}

This paper develops combined analytical and numerical tools to deal with static behavior and vibration frequencies of uniform elastic beams with uncertain rotational springs at the ends.

Interval uncertainty is dealt with a novel parameterization technique by introducing artificial parameter and a trigonometric function to describe uncertain variables. The advantageous characteristic of this study lies in overcoming the overestimation that is frequently characteristic to classical interval analysis.

It is hoped that the suggested methodology will be utilized in both identification of boundary conditions and in model updating.

\section{Acknowledgment}

This work was partially supported by South East Renewable Marine Energy Center (SERMEC). It was conducted when Mr. Clément Soret was on training program at the Florida Atlantic University's Department of Ocean and Mechanical Engineering. The partial support of CROUS (Centre Régional des Euvres Universitaires et Scolaires), Clermont-Ferrand, France and Conseil Régional d'Auvergne, Clermont-Ferrand, France, is gratefully appreciated.

\section{References}

[1] P.A.A. Laura and R.H. Guttierez, Vibrations of an elastically restrained cantilever beamof varying cross section with tip mass of finite length, Journal of Sound and Vibration 108(1) (1986), 123-132.

[2] M. Gürgöze, On the approximate determination of the fundamental frequency of a restrained cantilever beam carrying a tip heavy body, Journal of Sound and Vibration 105(3) (1986), 443-449.

[3] S.I. Alvarez, G.M. Ficcadenti de Iglesias and P.A.A. Laura, Vibrations of an elastically restrained non-uniform beam with translational and rotational springs, and with tip mass, Journal of Sound and Vibration 120(3) (1988), 465-471.

[4] A.L. Sweet, J. Genin and P.F. Mlakar, Vibratory identification of beam boundary conditions, Journal of Dynamic Systems Measurement and Control 98 (1976), 387-394.

[5] Y. Ben-Haim and A.G. Natke, Diagnosis of changes in elastic boundary conditions in beams by adaptive vibrational testing, Archive of Applied Mechanics 62(3) (1992), 210-221.

[6] I. Elishakoff and Y.Y. Fang, Diagnosis of local uncertain modifications in the boundary conditions of a rectangular plate via convex modeling, Computer Methods in Applied Mechanics and Engineering 124(4) (1995), 303-319.

[7] A. Cherki, G. Plessis, B. Lallemand, T. Tison and P. Level, Fuzzy behavior of mechanical systems with uncertain boundary conditions, Computer Methods, in Applied Mechanics and Engineering 189(3) (2000), 863-873.

[8] B.F. Zalewski, R.L. Mullen and R.L. Muhanna, Interval boundary element method in the presence of uncertain boundary conditions, integration errors, and truncation errors, Engineering Analysis with Boundary Elements 33(4) (2009), 508-513.

[9] S.S. Rao, Mechanical Vibrations, (5 ${ }^{\text {th }}$ edition), 725, Prentice Hall, Upper Saddle River, NJ (2011).

[10] T.C. Fung, Improved approximate formulas for the natural frequencies of simply supported Bernouilli-Euler beams with rotational restrains at the ends, Journal of Sound and Vibration 273 (2004), 451-455.

[11] I. Elishakoff, Bolotin's dynamic edge effect method, Shock and Vibration Digest 8(1) (1976), 95-104.

[12] D.B. Xiu and G.E. Karniadakis, Supersensitivity due to uncertain boundary conditions, International Journal for Numerical Methods in Engineering 61(2) (2004), 2114-2138. 

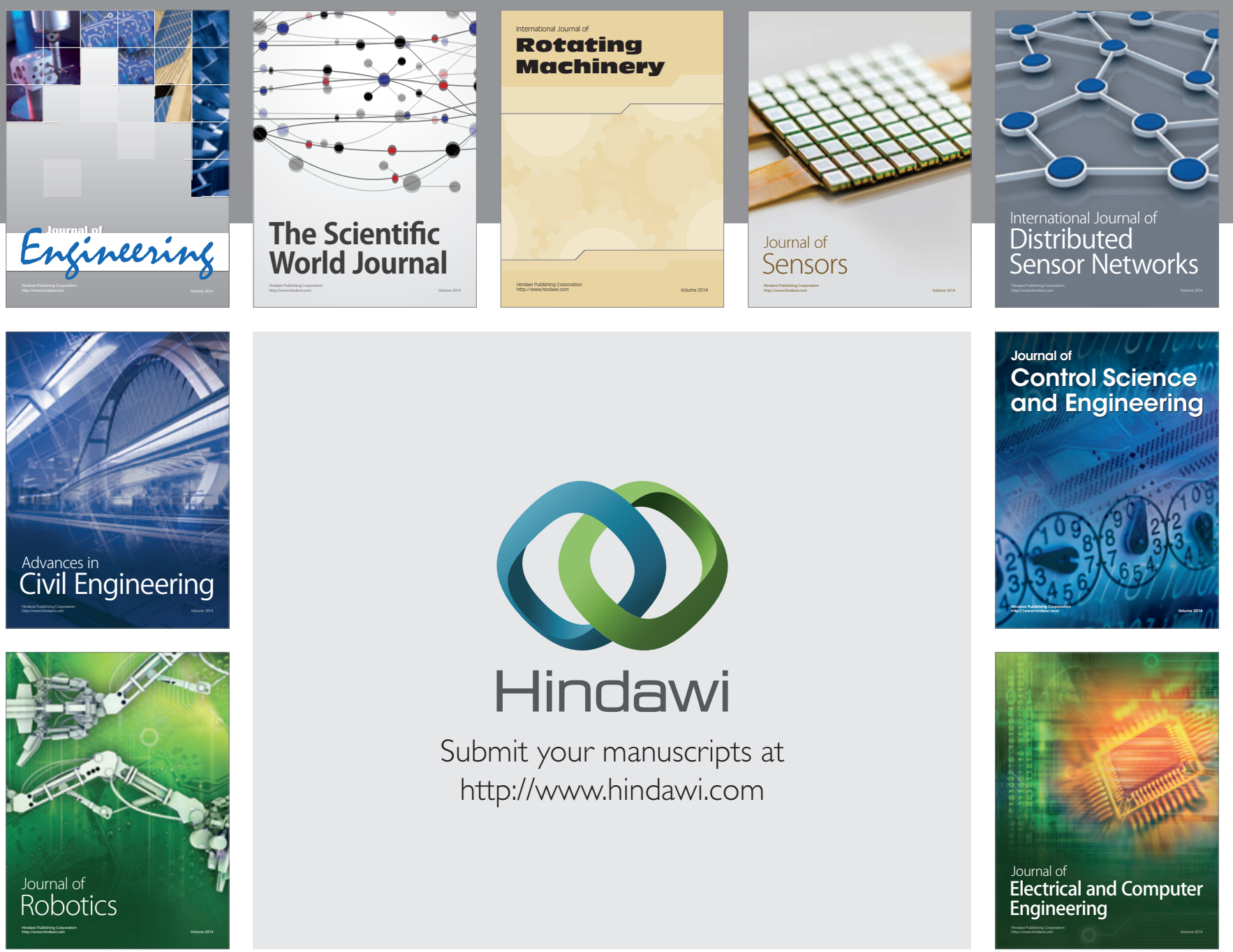

Submit your manuscripts at

http://www.hindawi.com
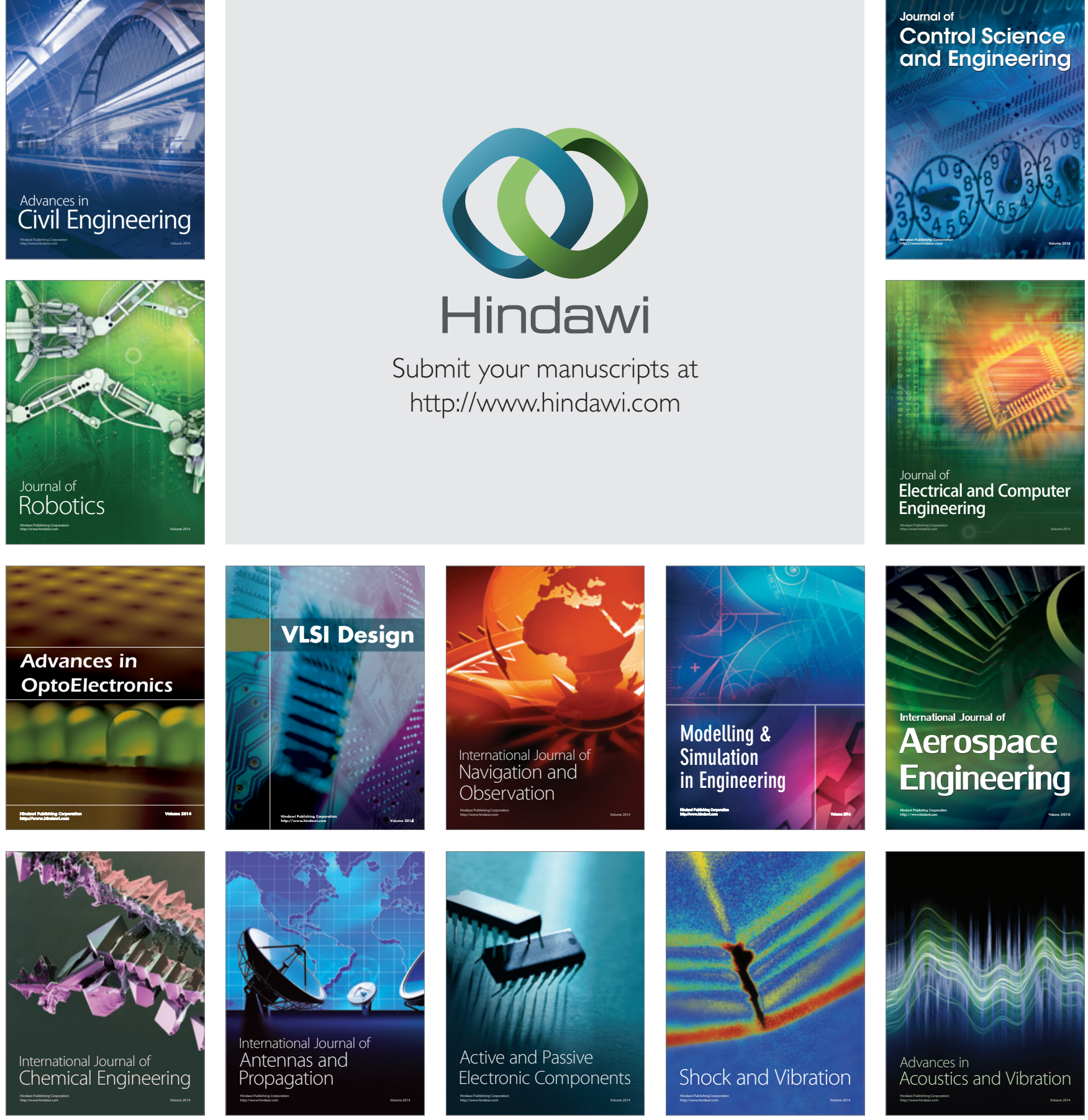